\title{
Editorial Preface to the Special Issue on Ubiquitous Computing Systems V
}

\author{
YASUYUKI SUMI $^{1, a)}$
}

Ubiquitous computing is research area aimed to enrich our daily lives and social activities using information technologies, and interdisciplinary area combined with $\mathrm{I} / \mathrm{O}$ devices, sensing, networking, middleware, human-interface, etc. Psychology and sociology are also important parts since ubiquitous computing systems should be used and evaluated in the context of human/social activities.

The papers submitted to the special issue cover various directions, and show common issues such as integration and scalingup of multiple technologies; project management from problem finding to problem solving; and ongoing evaluation of proposed systems embedded in the real world.

The issue has accepted 13 papers out of 19 submissions. The accepted papers cover wide area of ubiquitous computing technologies such as sensor networking, mobile sensing using smartphones, information recommendation, and application development in real fields such as disaster countermeasures, nursing care.

We have a succeeding issue on ubiquitous computing systems (submission due: December 9th, 2016). You can find the call for papers at http://www.ipsj.or.jp/english/jip/cfp/17-W.html.

I would like to thank my colleagues for their excellent work on reviewing. Without their affectionate and devoted work between authors and reviewers, such high acceptance rate as $68 \%$ was not realized. I'm greatly proud of the committee members.
The Editorial Committee

- Editor in-Chief:

Yasuyuki Sumi (Future University Hakodate)

- Editorial Board:

Sozo Inoue (Kyushu Institute of Technology)

Jin Nakazawa (Keio University)

\section{- Editorial Committee:}

Kazushige Ouchi (TOSHIBA Corporation)

Ren Ohmura (Toyohashi University of Technology)

Kaori Fujinami (Tokyo University of Agriculture and Technology)

Takeshi Iwamoto (Toyama Prefectural University)

Keisuke Uehara (Keio University)

Yu Enokibori (Nagoya University)

Yoshihiro Kawahara (The University of Tokyo)

Yasue Kishino (NTT)

Arei Kobayashi (KDDI R\&D Laboratories)

Ichiro Siio (Ochanomizu University)

Midori Sugaya (Shibaura Institute of Technology)

Masatoshi Sekine (Oki Electric Industry Co., Ltd.)

Hideyuki Takahashi (Tohoku University)

Emi Tamaki (Waseda University)

Koji Tsukada (Future University Hakodate)

Hitomi Tsujita (SyncPhase)

Takayuki Nakamura (NTT)

Nobuhiko Nishio (Ritsumeikan University)

Yasutaka Nishimura (KDDI R\&D Laboratories)

Takuya Maekawa (Osaka University)

Keiichi Yasumoto (Nara Institute of Science and Technology)

Kohei Matsumura (Ritsumeikan University)

Koji Yatani (The University of Tokyo)

Kazunori Yamada (Panasonic)

Takuro Yonezawa (Keio University)

Masahiro Toyoura (Yamanashi University) 